\title{
The risk of cholelithiasis in patients after heart transplantation
}

\author{
Piotr Wegrzyn, Marcin Popiolek, Piotr Przybylowski, Karol Wierzbicki, Kornelia Zareba, \\ Irena Milaniak, Boguslaw Kapelak, Krzysztof Bartus, Roman Pfitzner, Jerzy Sadowski
}

Department of Cardiovascular Surgery and Transplantology, Institute of Cardiology, Jagiellonian University, John Paul II Hospital, Krakow, Poland

Submitted: 13 April 2012

Accepted: 1 December 2012

Arch Med Sci 2014; 10, 1: 53-57

DOI: $10.5114 /$ aoms.2014.40733

Copyright @ 2014 Termedia \& Banach

\section{Abstract}

Introduction: Extended immunosuppressive treatment in patients after heart transplantation modifies etiopathogenesis and occurrence of many diseases in this population. The aim of the present study was to evaluate the frequency and to define risk factors for cholelithiasis after heart transplantation (HTX)

Material and methods: The study population consisted of 176 subjects. Of them, 24 patients (group A) presented with symptomatic cholelithiasis. Another group of 24 patients without cholelithiasis (group B) served as controls. Both groups were similar with respect to age, gender and follow-up after the transplant. Clinical interview, surgical and hospitalization data were collected from medical records.

Results: The groups did not differ in demographic features. There were statistical differences $(p<0.05)$ between group A and B in rejection reaction, doses of immunosuppressive drugs, type 2 diabetes, serum lipid disorders and acute rejection episodes. These events were caused by modification of treatment, especially the immunosuppressive regimen. Group A consisted of $75 \%$ men and $25 \%$ women. The frequency of symptomatic cholelithiasis was $11.7 \%$ in men and $27.3 \%$ in women, on average $19.5 \%$. Mean time to cholelithiasis following HTX was $37.9 \pm 4.9(\mathrm{Me}=41.5)$ months, $27.7 \pm 8.2$ $(M e=30.0)$ months in women and $41.3 \pm 5.9(M e=41.5)$ months in men. The female to male ratio was $2.3: 1$.

Conclusions: Cholelithiasis following HTX was significantly more frequent as compared with the non-transplant population. Patients with cholelithiasis required more aggressive immunosuppression because of more frequent episodes of acute transplant rejection. Patients with cholelithiasis significantly more frequently showed increased glycemia and blood lipids, which could be the side effect of intensive immunosuppressive therapy.

Key words: transplantation, cholelithiasis, immunosuppression, cyclosporine A.

\section{Introduction}

Heart transplantation (HTX) nowadays is still the most effective treatment of patients with end-stage heart failure with a "bad prognosis" [1-3]. Management of the patient after heart transplantation is a challenge and requires collaboration between physicians of different specialties. Immunosuppressants in high doses and their side-effects amplify the effects of coexistent risk factors such as cholelithiasis, nephrolithi-

\author{
Corresponding author: \\ Piotr Wegrzyn MD \\ Department \\ of Cardiovascular Surgery \\ and Transplantology \\ Institute of Cardiology \\ Jagiellonian University \\ John Paul II Hospital \\ 80 Pradnicka St \\ 31-202 Krakow, Poland \\ Phone: +48 126143075 \\ Fax: + 48124233900 \\ E-mail: peter-wegrzyn@o2.pl
}


asis, diabetes, atherosclerosis, deep vein thrombosis and others [4]. Increased morbidity due to cholelithiasis observed in patients following HTX probably results from disadvantageous effects of immunosuppressive treatment in those patients who require higher doses of medicines because of the severity of transplant rejection. Decreased immunity in these patients increases the likelihood of acute septic complications of cholelithiasis, shifting the patients to a high-risk group characterized by a particularly high probability of complication and death.

The aim of the study is to evaluate the frequency of cholelithiasis and to define risk factors for developing cholelithiasis after HTX.

\section{Material and methods}

\section{Patient population}

A group of 176 patients ( $M=154$ (87.5\%), $F=22$ $(12.5 \%))$, over 40 years of age, operated on between 1996 and 2005, was identified. Of them, 24 with symptomatic cholelithiasis were included in the retrospective analysis (group A). Patients operated on before the year 1996 were excluded from the analysis because of incomplete data (abdominal ultrasound, echo data and details of immunosuppression protocols). The patients in group A did not have significant gallstones before heart transplantation - data confirmed by abdominal USG done routinely before HTX. Another group of 24 patients without cholelithiasis (group B) served as controls. Both groups were similar with respect to age, gender and follow-up after the transplant. Abdominal ultrasound was used to confirm (group A) or exclude (group B) the presence of cholelithiasis. Clinical interview, surgical and hospitalization data were collected from medical records.

\section{Patient management}

All patients in both groups were treated with cyclosporine, and either azathioprine or mycophenolate mofetil. All doses of medicines and administration of azathioprine (AZA) or mycophenolate mofetil (MMF) were tailored for each patient depending on the severity of transplant rejection. Steroids were given transiently in case of acute rejection and clinical deterioration of the patient.

\section{Statistical analysis}

Groups A and B were compared with respect to body mass index (BMI), coexisting lipid disorders, type 2 diabetes, average immunosuppressant doses (cyclosporine, MMF, AZA), as well as the number and transplant rejection grade. There were no cases of type 1 diabetes.
All data are presented as means \pm SE (standard error) unless otherwise indicated. For comparisons between group $A$ and $B$ of the crosssectional study, the Shapiro-Wilk test was performed (for estimating normal distribution), then either Student's t-test (for equal variances) or Cochran-Cox's test (for different variances) was used for data analysis. Homogeneity of variance was checked by Levene's test. The $\chi^{2}$ test was used for analysis of BMI and the Kruskal-Wallis test for multivariable analysis of ISHLT grade. Standard deviation (SD) and median (Me) were also calculated. All statistical analyses were performed using the statistical software package Statistica (StatSoft, Inc. Tulsa, Oklahoma). We also used the Kaplan-Meier curve to analyze the long-term survival and log-rank test for comparing survival differences between groups of patients.

\section{Results}

\section{General data}

In group $A$, there were 16 men and 6 women $(M: F=3: 1)$. The average age was 56 years. Symptomatic cholelithiasis was observed in $27.3 \%$ of women ( 6 cases out of 22 ) and in $11.7 \%$ of men (18 cases out of 154), thus 2.3 times more often in women than in men. The average rate of cholelithiasis was $19.5 \%$. Mean time to cholelithiasis following HTX was $37.9 \pm 4.9$ ( $\mathrm{Me}=41.5$ ) months, $27.7 \pm 8.2(\mathrm{Me}=30.0)$ months in women and $41.3 \pm 5.9(\mathrm{Me}=41.5)$ months in men.

\section{Symptomatology}

The main symptom of cholelithiasis was biliary colic, which was observed in $79.2 \%$ of patients (19 out of 24), all of them being women in group A. Dyspepsia, defined as a "small symptom of cholelithiasis" was reported by $45.8 \%$ of patients (11 out of 24), but complications of cholelithiasis developed in about $33.3 \%$ of patients (8 out of 24 ). The complications were 3 times more frequent in women than in men, i.e. $66.7 \%$ ( 4 of 6 ) vs. $22.2 \%$ (4 of 18). In all patients in the study group, at least 2 out of 3 symptoms appeared. All patients with complications underwent cholecystectomy.

\section{Cholecystectomy}

Generally, $45.8 \%$ (11 of 24) of patients in the study group underwent cholecystectomy, including $66.7 \%$ of women (4 of 6 ) and $38.9 \%$ of men (7 of 18). Conventional and laparoscopic cholecystectomy was performed in $8(62.7 \%)$ and 3 patients $(27.3 \%)$, respectively. Mean time from transplantation to cholecystectomy was $42.6 \pm 8.7$ $(M e=38.0)$ months. In women, this interval was 
A Table I. Cholelithiasis risk factors

\begin{tabular}{|lccccccc|}
\hline Group & \multicolumn{3}{c}{ Body mass index } & \multicolumn{5}{c|}{ Lipid disturbances } \\
\cline { 2 - 8 } & Norm & Overweight & Obesity & HC $<1$ year & HTG $<1$ year & HC $>1$ year & HTG $>1$ year \\
\hline A & $3 / 24$ & $17 / 24$ & $4 / 24$ & $7 / 24$ & $6 / 24$ & $17 / 24$ & $16 / 24$ \\
& $(11.7 \%)$ & $(70.8 \%)$ & $(16.7 \%)$ & $(29.2 \%)$ & $(25.0 \%)$ & $(70.8 \%)$ & $(66.7 \%)$ \\
\hline B & $11 / 24$ & $12 / 24$ & $1 / 24$ & $5 / 24$ & $7 / 24$ & $5 / 24$ & $7 / 24$ \\
& $(45.4 \%)$ & $(50.0 \%)$ & $(4.2 \%)$ & $(20.8 \%)$ & $(29.2 \%)$ & $(20.8 \%)$ & $(29.2 \%)$ \\
\hline Value of $p$ & 0.01 & 0.76 & 0.16 & 0.51 & 0.75 & 0.002 & 0.009 \\
\hline
\end{tabular}

HC-hypercholesterolemia, HTG - hypertriglyceridemia

B

\begin{tabular}{|lcccccc|}
\hline Group & $\begin{array}{c}\text { Diabetes } \\
\text { type } 2\end{array}$ & $\begin{array}{c}\text { ISHLT } \\
\text { Gr }<1 \text { year }\end{array}$ & $\begin{array}{c}\text { ISHLT } \\
\text { Gr }>1 \text { year }\end{array}$ & CSA (SD) & MMF (SD) & AZA (SD) \\
\hline A & $3 / 24$ & $0-11 / 24(45.4 \%)$ & $0-11 / 24(45.4 \%)$ & $3.17 \pm 0.36$ & $28.9 \pm 17.3$ & $1.083 \pm 0.18$ \\
& $(11.7 \%)$ & $1 \mathrm{~A}-10 / 24(41.7 \%)$ & $\begin{array}{c}1 \mathrm{~A}-8 / 24(33.3 \%) \\
1 \mathrm{~B}-4 / 24(16.7 \%)\end{array}$ & & & \\
& & $3 \mathrm{~A}-3 / 24(11.7 \%)$ & $2 \mathrm{~A}-1 / 24(4.2 \%)$ & & & \\
\hline $\mathrm{B}$ & $1 / 24$ & $0-17 / 24(70.8 \%)$ & $0-24 / 24(100 \%)$ & $2.45 \pm 0.54$ & $23.5 \pm 3.3$ & $0.95 \pm 0.1$ \\
& $(4.2 \%)$ & $1 \mathrm{~A}-4 / 24(16.7 \%)$ & & & \\
& & $1 \mathrm{~B}-3 / 24(11.7 \%)$ & & & & \\
\hline Value of $p$ & 0.30 & 0.19 & $<0.001$ & $<0.001$ & 0.49 & 0.039 \\
\hline
\end{tabular}

ISHLT Gr - The International Society for Heart and Lung Transplantation System for Grading Rejection, CsA - cyclosporine A average doses (mg/kg b.m.), MMF - mycophenolate mofetil average doses ( $\mathrm{mg} / \mathrm{kg}$ b.m.), AZA - azathioprine average doses (mg/kg b.m.)

two times shorter $(24.5 \pm 10.6$ months $)(\mathrm{Me}=23.0)$ than in men ( $53 \pm 10.9$ months) $(\mathrm{Me}=46.0)$ ( Tables $\mid \mathrm{A}$ and $\mathrm{B})$.

Based on our observations, MMF instead of AZA effectively lowered inflammatory reactions in patients after HTX (group A). Mycophenolate mofetil allowed for use of a statistically lower ( $p=0.024$ ) dose of cyclosporine (average: 2.93 $\pm 0.35 \mathrm{mg} / \mathrm{kg}$ b.m.) when administered in combination with MMF. The mean dose of cyclosporine in connection with AZA was higher (average: $3.41 \pm 0.45 \mathrm{mg} / \mathrm{kg}$ b.m.).

With time, azathioprine was replaced by mycophenolate mofetil, resulting in the lowering of cyclosporine A (CsA) doses [5-10].

\section{Long-term survival}

Patients were followed up for 16 years after heart transplant. At the same time of observation a group of patients with cholelithiasis revealed a worse survival rate. Fourteen of 24 (58.3\%) patients survived in comparison to the control group in which 16 of 24 (66.6\%) patients survived. There was no statistically significant difference between A and B ( $p=0.37)$ (Figure 1).

\section{Discussion}

In the study group, the increased frequency of symptomatic cholelithiasis after HTX (19.5\% on average vs. $2.4 \%$ in general population) was probably associated with high doses of immu- nosuppressants - CsA and AZA [11]. The complex of cyclosporine binding with cyclophilin decreases the activity of $\mathrm{T}$ cells by inhibiting calcineurin, which is responsible for activation of interleukin 2. The reduced level of IL-2 leads to deteriorating function of T cells. Azathioprine is a pro-drug for 6-mercaptopurine. It blocks purine metabolism and DNA synthesis and strongly affects T and B proliferating cells. Immunosuppressants increased the risk of cholelithiasis, which developed more frequently in these patients as compared with the general population. The

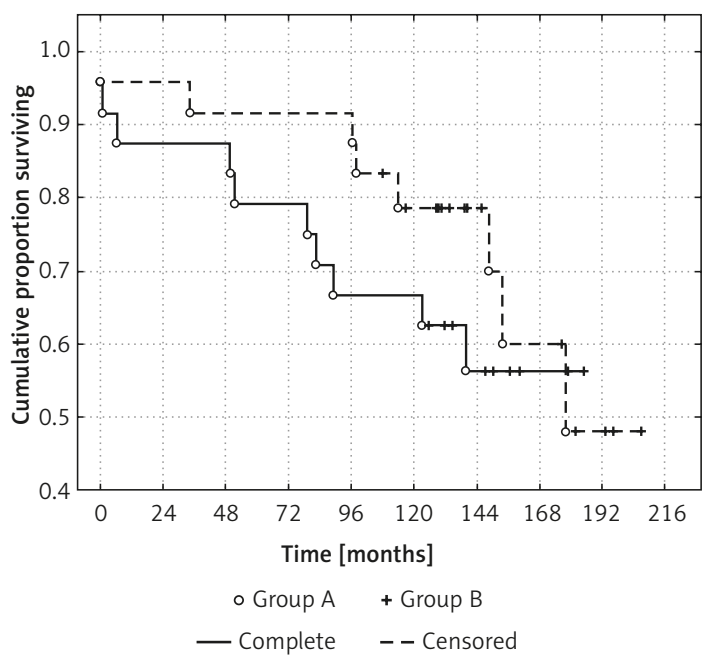

Figure 1. Kaplan-Meier curve: long-term surviving proportion between group A and group B. Log-rank test statistic $=-0.905054, p=0.36544$ 
following risk factors were statistically significant in group A: gender (2.3 times more frequent in women), obesity, diabetes mellitus type 2 and lipid disorders (the higher frequency of lipid disorders at long-term follow-up in group A was probably associated with the adverse side effects of immunosuppressants), higher rejection grade according to the ISHLT scale, and a necessity of using higher doses of CSA and AZA.

Prolonged immunosuppression after HTX is associated with progression of risk factors, which intensify the process of cholelithiasis [12]. Cholelithiasis could escalate in an immune response and inflammatory state, thus intensifying the transplant rejection.

Available evidence [13] shows that administration of MMF instead of AZA significantly reduces the inflammatory state in patients after HTX. Mycophenolate mofetil is a reversible inhibitor of monophosphate dehydrogenase (IMPDH). This enzyme is important in purine biosynthesis and maturation of T cells. Mycophenolate mofetil does not cause chromosomal aberrations, and thus shows a less mutagenic effect in comparison to azathioprine [14]. Mycophenolate mofetil generates less frequent side effects. Administration of MMF provides a possibility of reducing cyclosporine doses, which probably delays the process of cholelithiasis. Therefore, there is a probability that in patients with active cholelithiasis, treated with MMF, the risk of acute transplant rejection can be diminished through reducing the inflammatory state (decreasing the unfavorable positive feedback effect).

On the other hand, immunosuppressive agents used to suppress the inflammatory response, due to reduction in the number of resistant cells (the side effect of immunosuppression), contribute to the masking of the presence of the inflammatory process in patients with active cholelithiasis. It poses a serious risk of diagnosing the disease at a late stage. Furthermore, cholelithiasis in the general population is associated with risk of gallbladder cancer. Neoplasm in patients after organ transplantation who are on immunosuppression is one of the most frequent causes of death [15].

The present study suggests that patients after HTX should be monitored for the presence of biliary tract disease. Regular clinical examinations, abdominal ultrasound, and laboratory tests (CRP, ESR, leukocytosis) as a standard procedure allow for early diagnosis and avoidance of further complications [16-20]. This could increase long-term survival and potentially improve the quality of life [21] in patients after heart transplantation.

\section{Acknowledgments}

We are grateful to Prof. Anetta Undas MD, PhD for critical remarks and scientific support.

\section{References}

1. Task Force Members, McMurray JJ, Adamopoulos S, Anker SD, et al. ESC Guidelines for the diagnosis and treatment of acute and chronic heart failure 2012: The Task Force for the Diagnosis and Treatment of Acute and Chronic Heart Failure 2012 of the European Society of Cardiology. Developed in collaboration with the Heart Failure Association (HFA) of the ESC. Eur Heart J 2012; 33:1787-847.

2. Feola M, Lombardo E, Testa M, et al. Prognostic factors of mid-term clinical outcome in congestive heart failure patients discharged after acute decompensation. Arch Med Sci 2012; 8: 462-70.

3. Sekhri V, Sanal S, DeLorenzo L, et al. Cardiac sarcoidosis: a comprehensive review. Arch Med Sci 2011; 7: 546-54.

4. Aliabadi A, Cochrane AB, Zuckermann AO. Current strategies and future trends in immunosuppression after heart transplantation. Curr Opin Organ Transplant 2012; 17: 540-5.

5. Lord RV, Ho S, Coleman MJ, et al. Cholecystectomy in cardiothoracic organ transplant recipients. Arch Surg 1998; 133: 73-9.

6. Richardson WS, Surowiec WJ, Carter KM, et al. Gallstone disease in heart transplant recipients. Ann Surg 2003; 237: 273-6.

7. Takeyama H, Sinanan MN, Fishbein DP, et al. Expectant management is safe for cholelithiasis after heart transplant. J Heart Lung Transplant 2006; 25: 539-43.

8. Englesbe MJ, Dubay DA, Wu AH, et al. Gallbladder disease in cardiac transplant patients: a survey study. Arch Surg 2005; 140: 399-403.

9. Barten MJ, Rahmel A, Boldt A, et al. Pharmacodynamic monitoring of the immunosuppressive therapy in patients after heart transplantation: whole blood flow cytometric analysis of lymphocyte function. Comput Biol Med 2007; 37: 1367-73.

10. Bączkowska T, Durlik M. Calcineurin inhibitor sparing immunosuppressive regimens in kidney allograft recipients. Pol Arch Med Wewn 2009; 119: 318-25.

11. Garlicki M, Czub P, Labus K, et al. Conversion from cyclosporine to tacrolimus improves renal function and lipid profile after cardiac transplantation. Ann Transplant 2006; 11: 24-7.

12. Barten MJ, Rahmel A, Garbade J, et al. Pharmacodynamic monitoring of the conversion of cyclosporine to tacrolimus in heart and lung transplant recipients. Transplant Proc 2005; 37: 4532-4.

13. White $M$, Haddad H, Leblanc MH, et al. Conversion from cyclosporine microemulsion to tacrolimus-based immunoprophylaxis improves cholesterol profile in heart transplant recipients with treated but persistent dyslipidemia: the Canadian multicentre randomized trial of tacrolimus vs cyclosporine microemulsion. J Heart Lung Transplant 2005; 24: 798-809.

14. Rowiński W, Wałaszewski J, Pączka L. Transplantologia kliniczna [Polish]. Wydawnictwo Lekarskie PZWL, Warsaw, 2004; 144-5.

15. Bhatia D, Bowen J, Money S, et al. The incidence, morbidity, and mortality of surgical procedures after orthotopic heart transplantation. Ann Surg 1997; 225: 686-94. 
16. Pethig K, Heublein B, Wahlers T, et al. Mycophenolate mofetil for secondary prevention of cardiac allograft vasculopathy: influence on inflammation and progression of intimal hyperplasia. J Heart Lung Transplant 2004; 23: 61-6.

17. Szmidt J, Gruca Z, Krawczk M, et al. General. Surgery. Practical Medicine 2003.

18. Halloran PF, Miller LW. In vivo immunosuppressive mechanisms. J Heart Lung Transplant 1996; 15: 959-71.

19. Bateson MC. Gallbladder disease and cholecystectomy rate are independently variable. Lancet 1984; 15: 621-4.

20. Spes CH, Angermann CE, Beyer RW, et al. Increased incidence of cholelithiasis in heart transplant recipients receiving cyclosporine therapy. J Heart Transplant 1990; 9: 404-7.

21. Jaworska I, Pudlo R, Pacholewicz J, et al. Return to life and the quality of life assessment after hart transplantation. Kardiochir Torakochi 2011; 8: 153-6. 